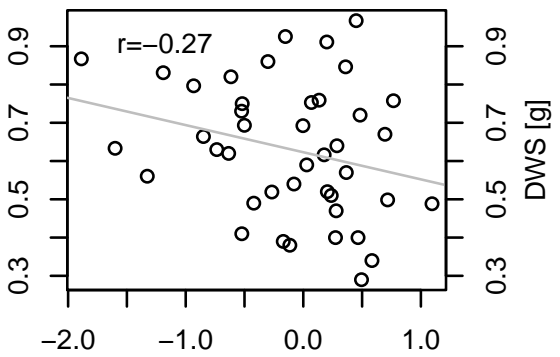

Relative Intensity [log10]

M2 Monomethylphosphate

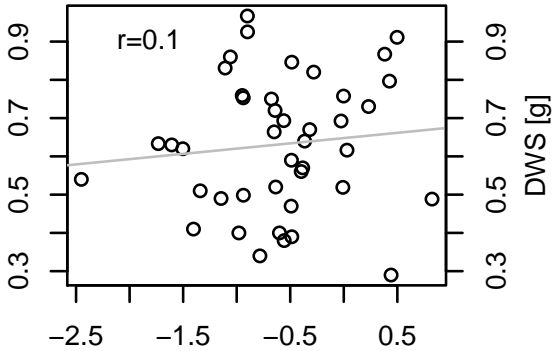

Relative Intensity [log10]

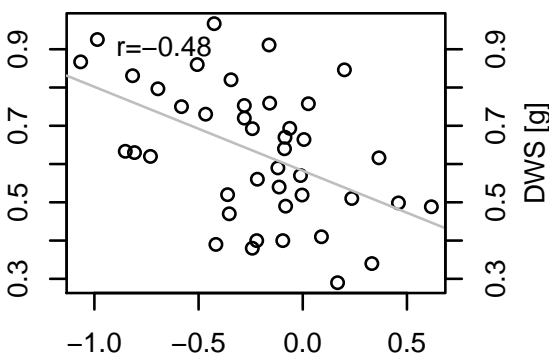

Relative Intensity [log10]

M5 Phosphoric acid

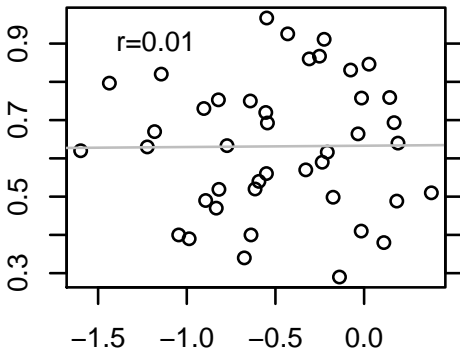

Relative Intensity [log10]

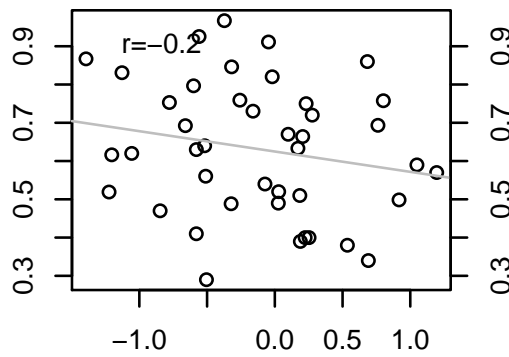

Relative Intensity [log10]
M8 Glyceric acid

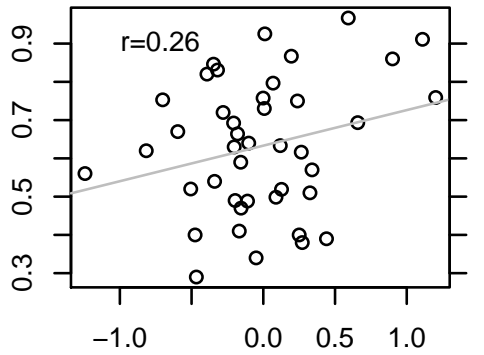

Relative Intensity [ $\log 10]$
M6 Glycine

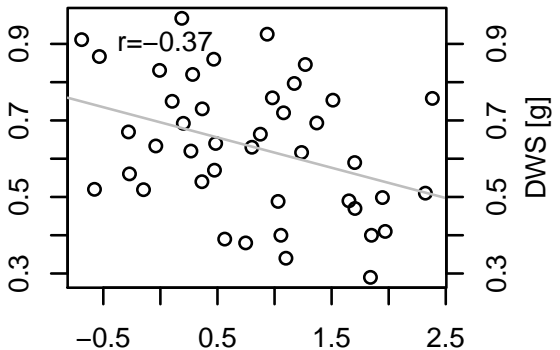

Relative Intensity [log10]

\section{M9 Uracil}

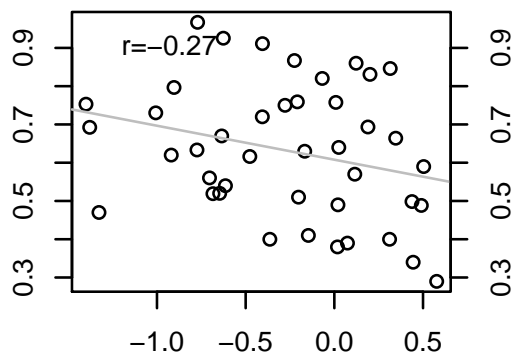

Relative Intensity [ $\log 10]$

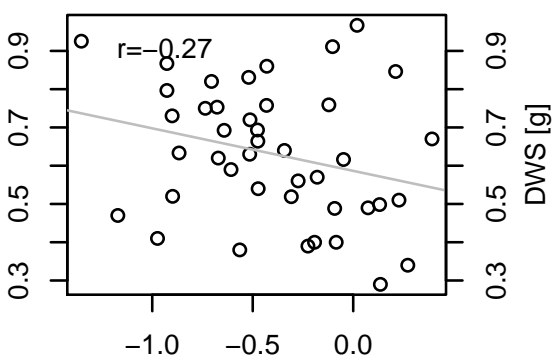

Relative Intensity [log10] 


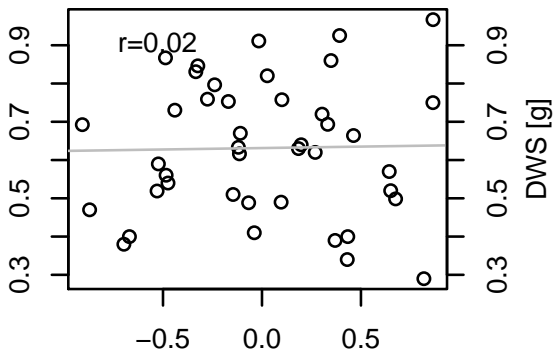

Relative Intensity [log10]

M11 Serine

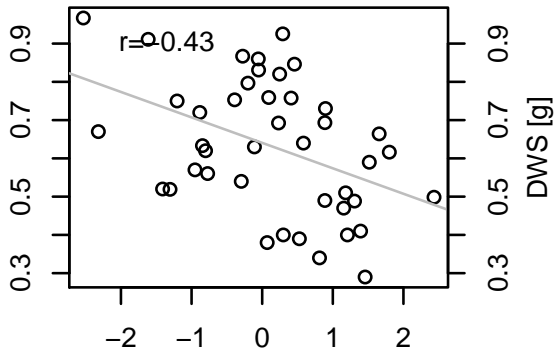

Relative Intensity [log10]

M12 Threonic acid-1,4-lactone

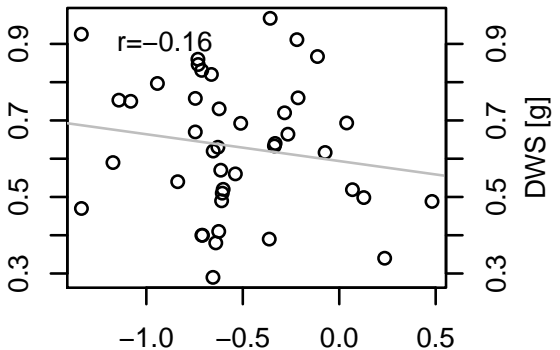

Relative Intensity [log10]

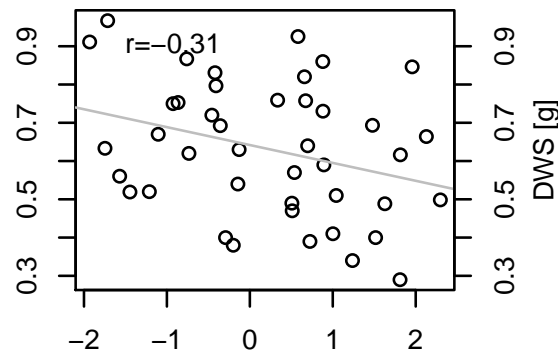

Relative Intensity [log10]

M14 MST 1419.8

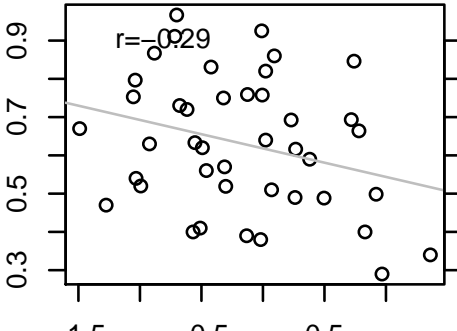

Relative Intensity [log10]

M15 Erythronic acid-1,4-lactone

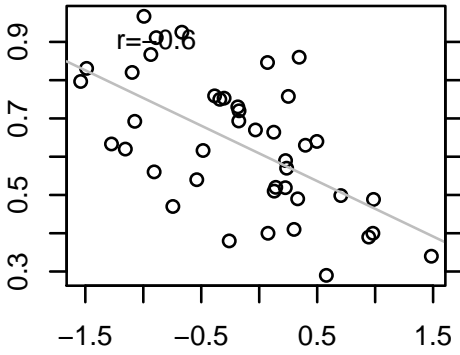

Relative Intensity [log10]

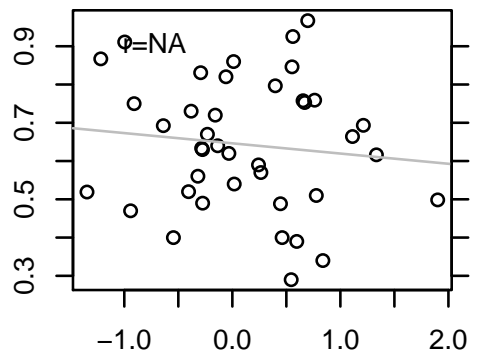

Relative Intensity [log10]

M17 Malic acid

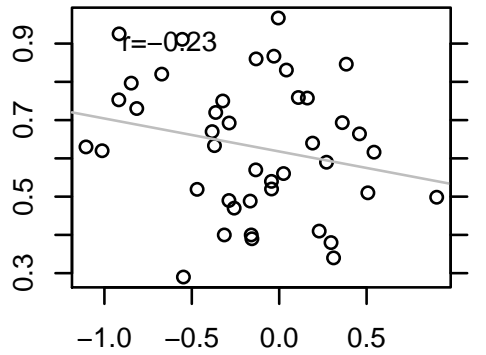

Relative Intensity [ $\log 10]$

M18 Erythritol

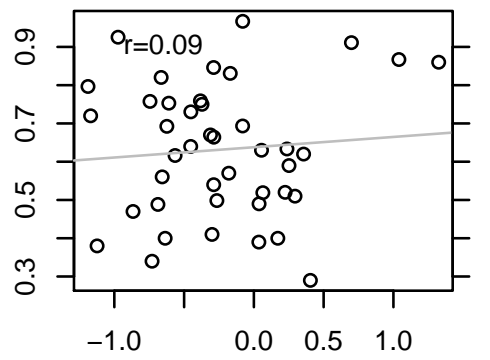

Relative Intensity [ $\log 10]$ 


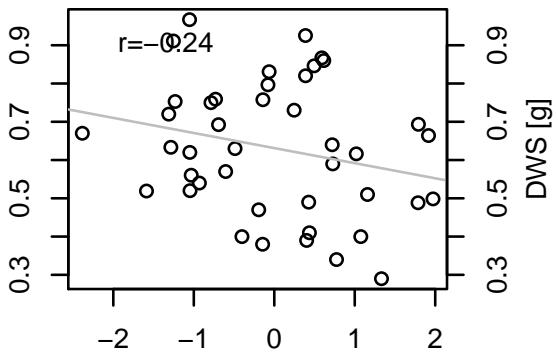

Relative Intensity [log10]

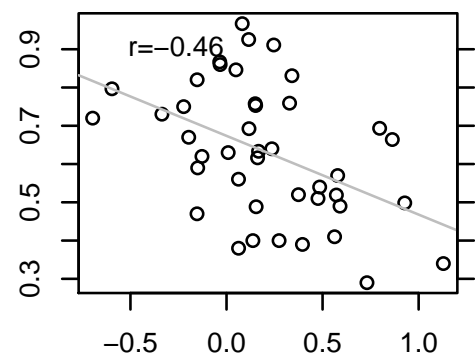

Relative Intensity [log10]

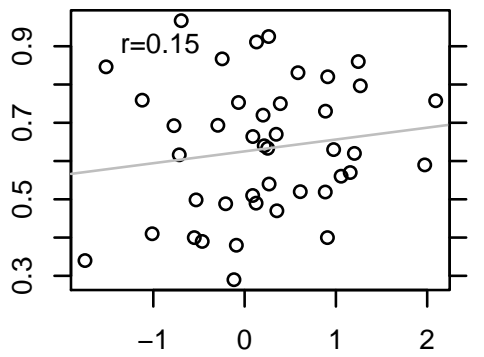

Relative Intensity $[\log 10]$
M20 Pyroglutamic acid (Glutamine, Glutam

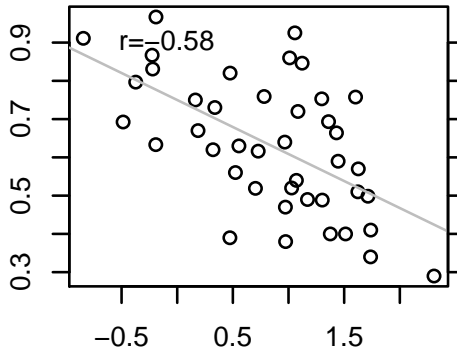

Relative Intensity [log10]

M21 Glutamic acid

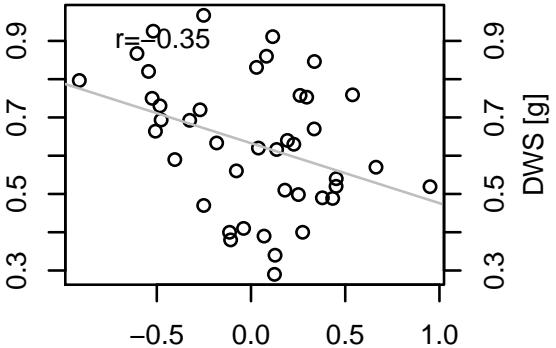

Relative Intensity [log10]
M23 Threonic acid

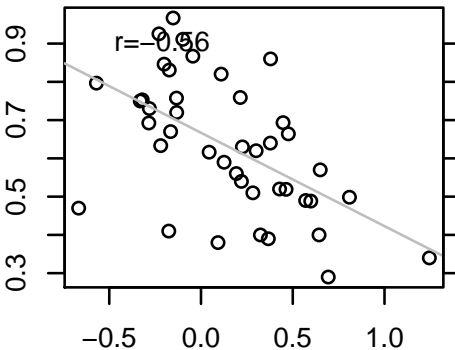

Relative Intensity [log10]

M24 Phenylalanine

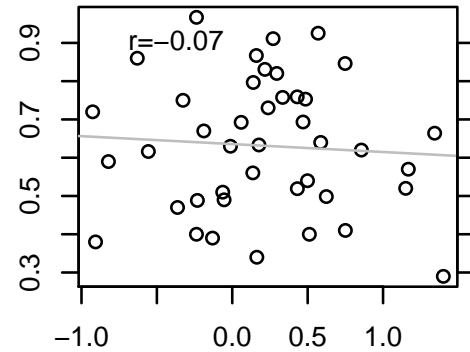

Relative Intensity [ $[\log 10]$
M26 MST 1574.3

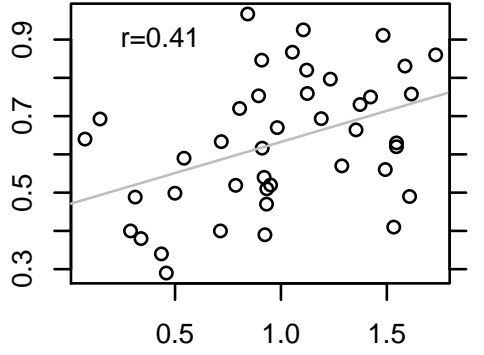

Relative Intensity [log10]

M27 Glutamic acid

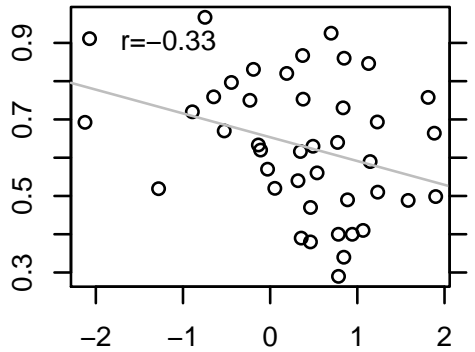

Relative Intensity $[\log 10]$ 


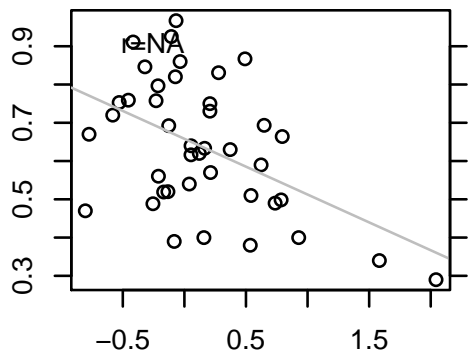

Relative Intensity [log10]

M29 Asparagine

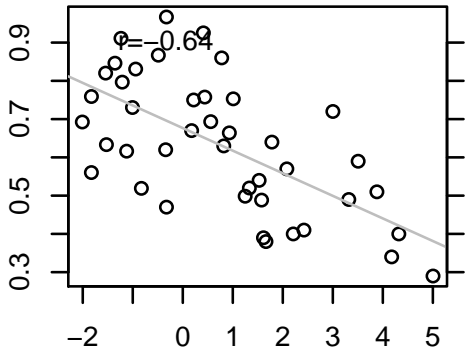

Relative Intensity [log10]

M30 Alcohol (Polyol, Pentahydroxy-)

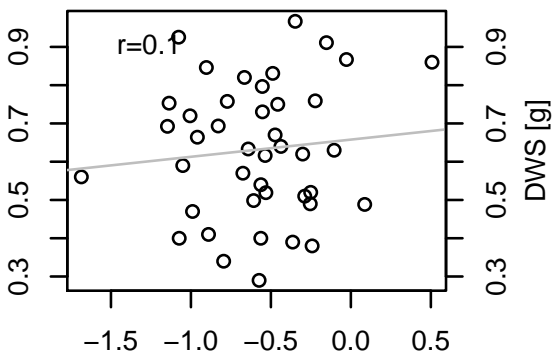

Relative Intensity [log10]
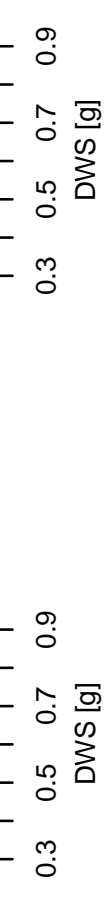

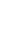

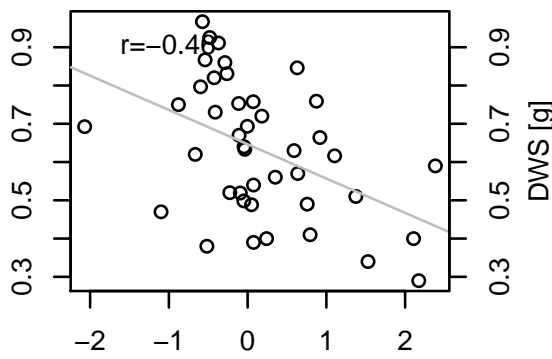

Relative Intensity [log10]

M32 1,6-Anhydro-beta-D-glucose

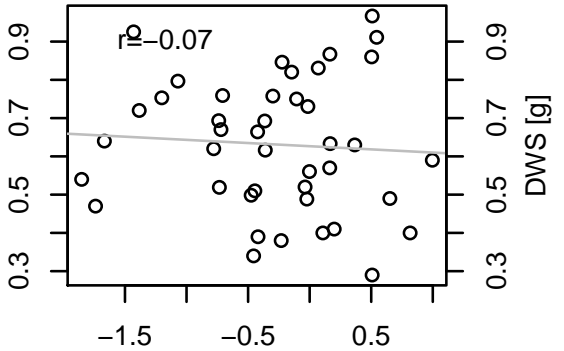

Relative Intensity [log10]

M33 Ribitol

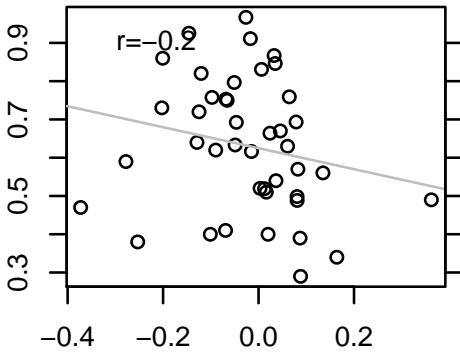

Relative Intensity [ $\log 10]$

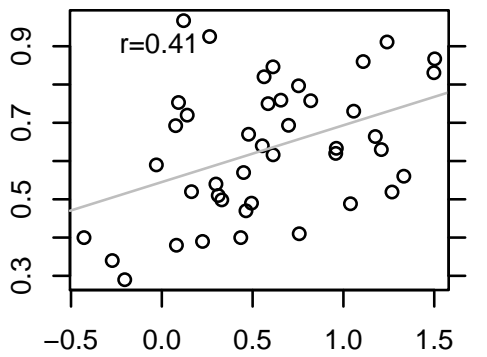

Relative Intensity [log10]

M35 MST 1732.9

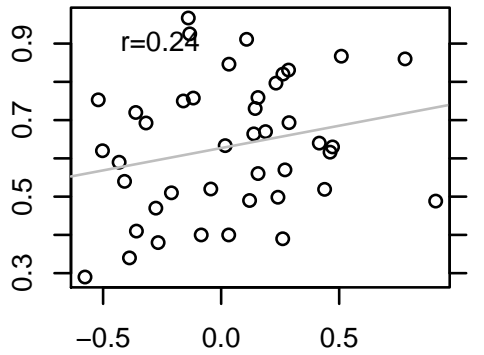

Relative Intensity [ $\log 10]$

M36 Putrescine

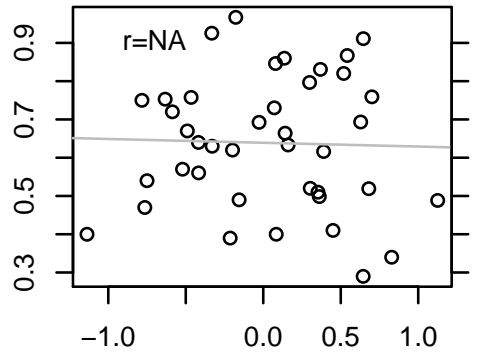

Relative Intensity [ $\log 10]$ 
M37 Ribonic acid

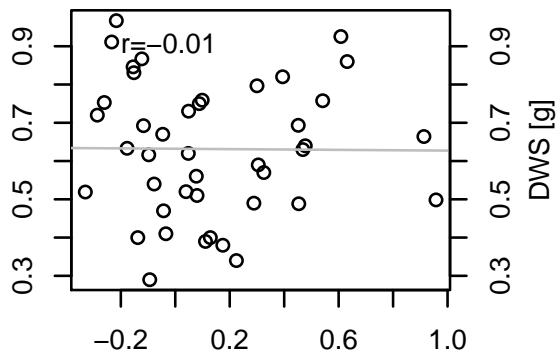

Relative Intensity [log10]

M38 Glycerol-3-phosphate

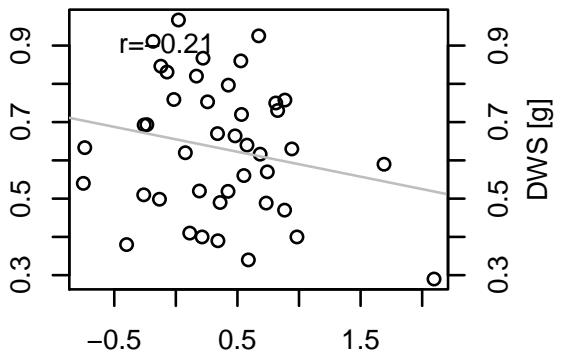

Relative Intensity [ $\log 10]$

M39 MST 1756.27

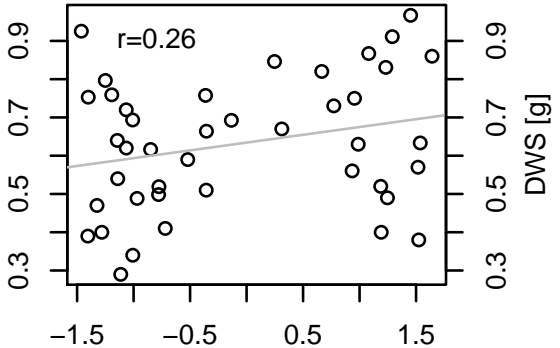

Relative Intensity [log10]

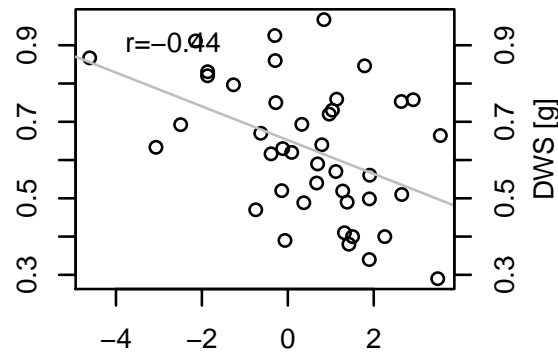

Relative Intensity [log10]

M41 Glyceric acid-3-phosphate

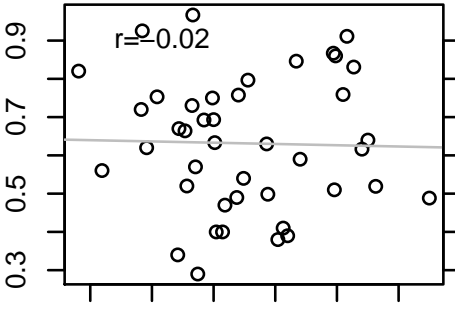

$\begin{array}{lll}-2.0 & -1.0 & 0.0\end{array}$

Relative Intensity [log10]

M42 Shikimic acid

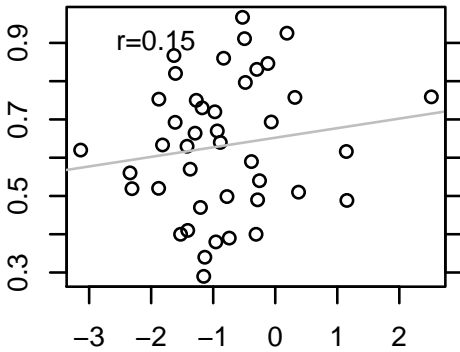

Relative Intensity [log10]
M43 Citric acid

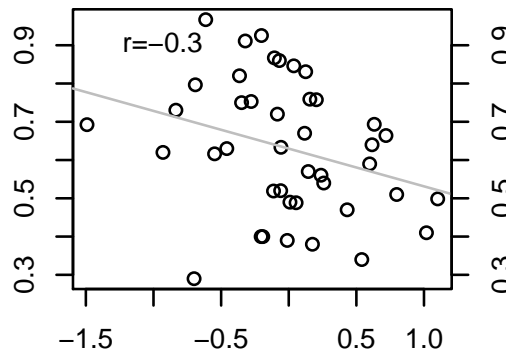

Relative Intensity [log10]

M44 MST 1816.2

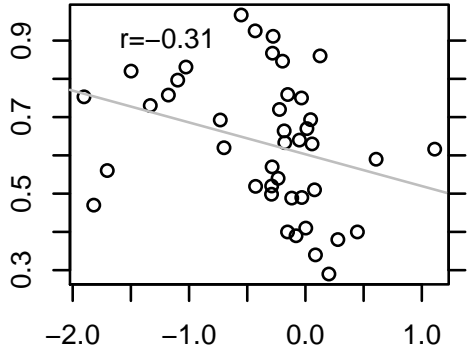

Relative Intensity [ $\log 10]$

M45 Quinic acid

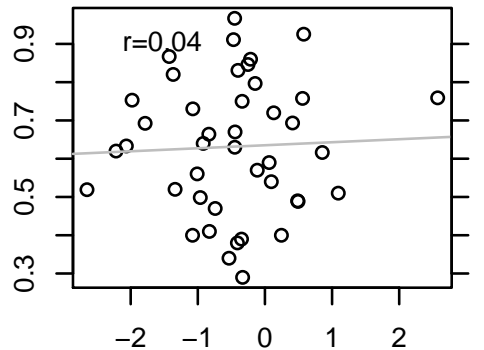

Relative Intensity $[\log 10]$ 


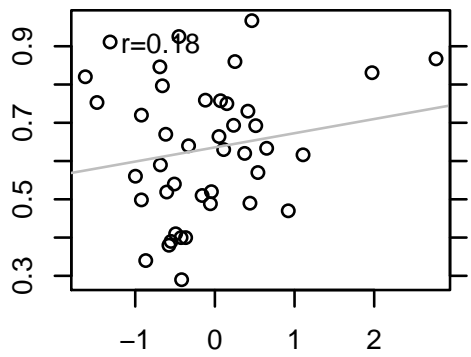

Relative Intensity [log10]

M47 Sorbose

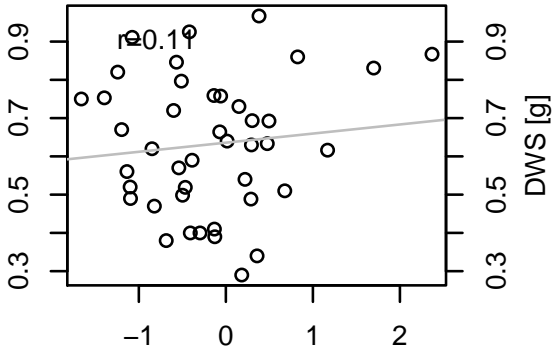

Relative Intensity [log10]

M48 Allantoin

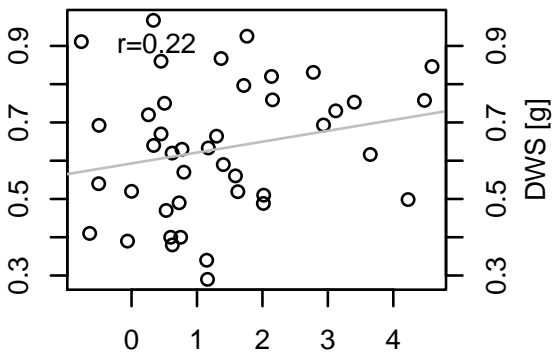

Relative Intensity [log10]

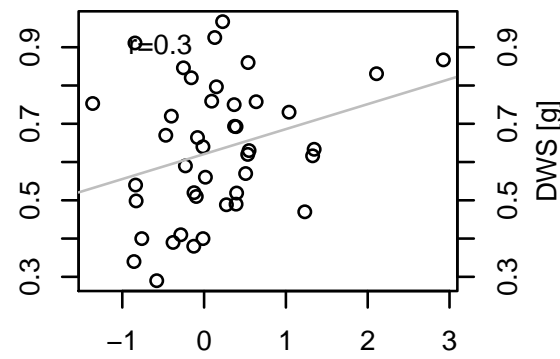

Relative Intensity [log10]

M50 Gluconic acid-1,4-lactone

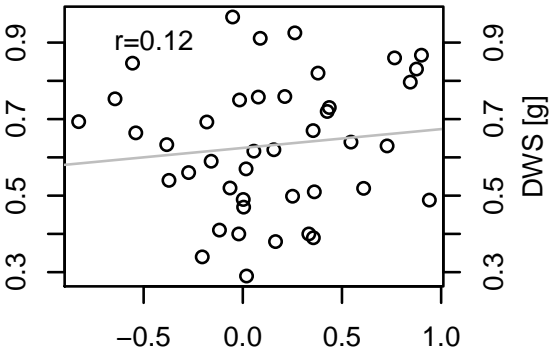

Relative Intensity [log10]

M51 Tyrosine

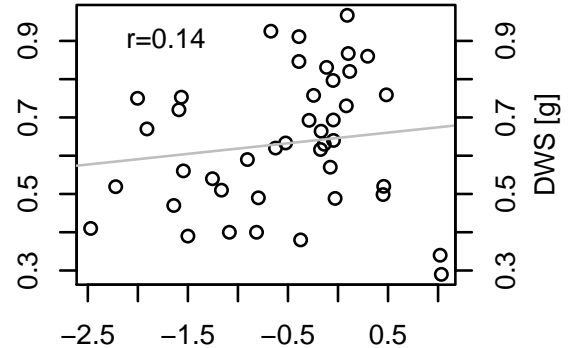

Relative Intensity [log10]

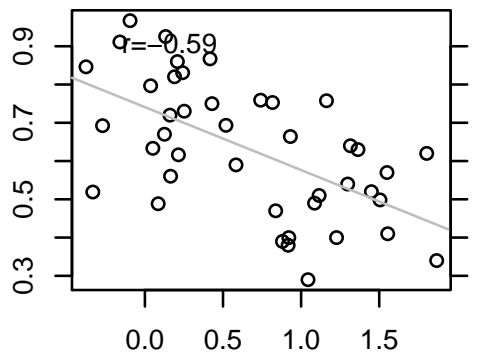

Relative Intensity [log10]

M53 Gluconic acid

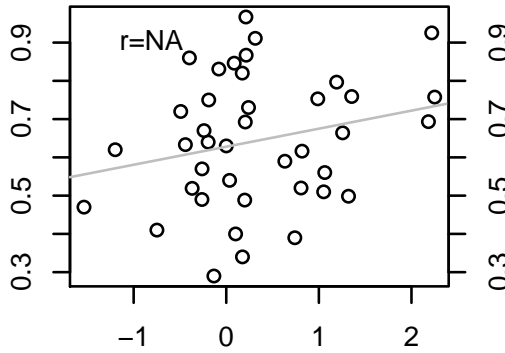

Relative Intensity [log10]

M54 MST 2002.33

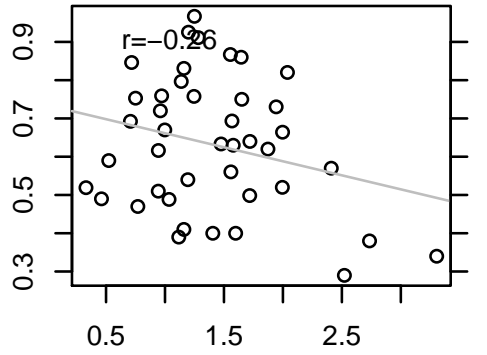

Relative Intensity [ $\log 10]$ 


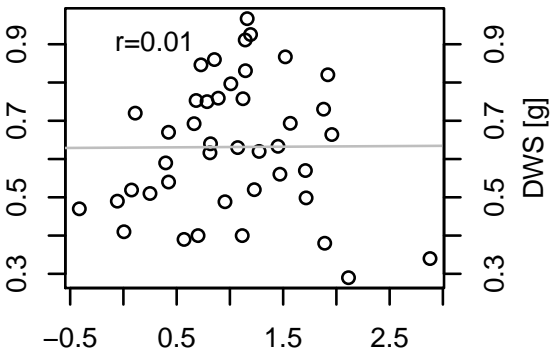

Relative Intensity [log10]

M56 Galactaric acid

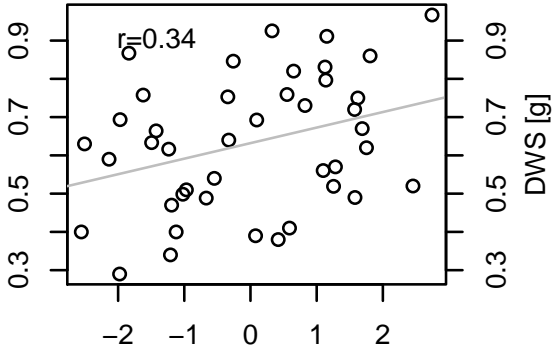

Relative Intensity [log10]

M57 myo-Inositol

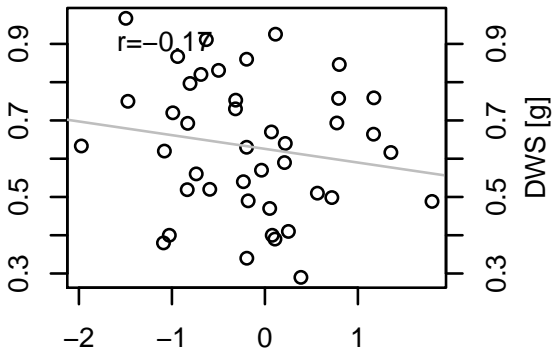

Relative Intensity [log10]

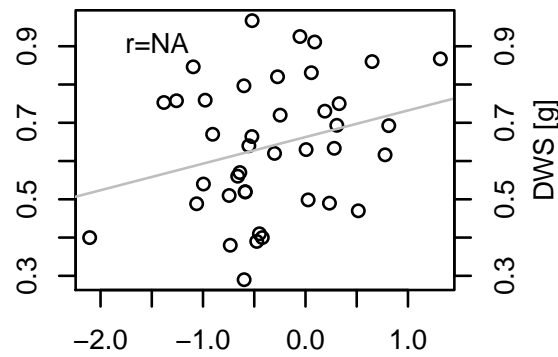

Relative Intensity [log10]

M59 MST 2160.33

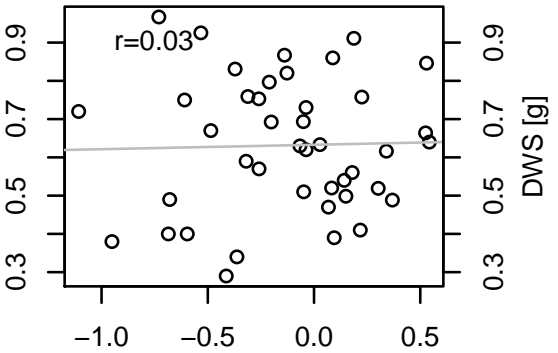

Relative Intensity [log10]

M60 Octadecanoic acid

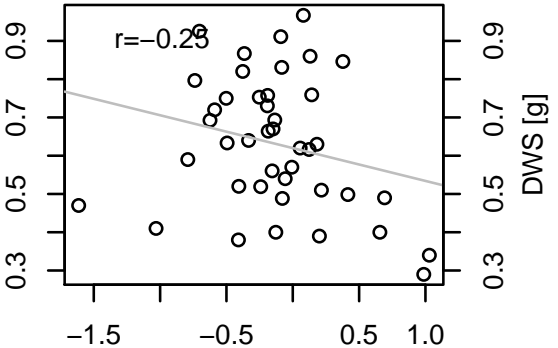

Relative Intensity [ $\log 10]$

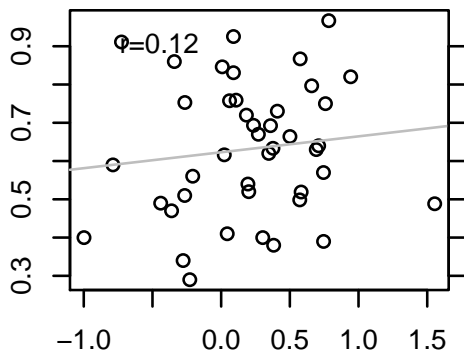

Relative Intensity [log10]

M62 Fructose-6-phosphate

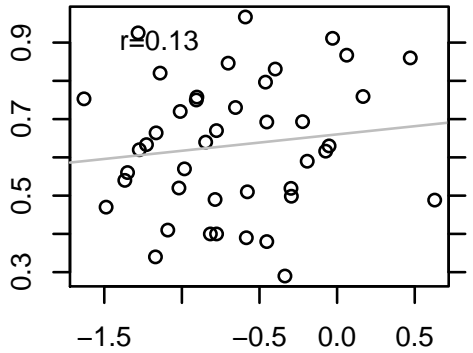

Relative Intensity [log10]

M63 Galactosylglycerol

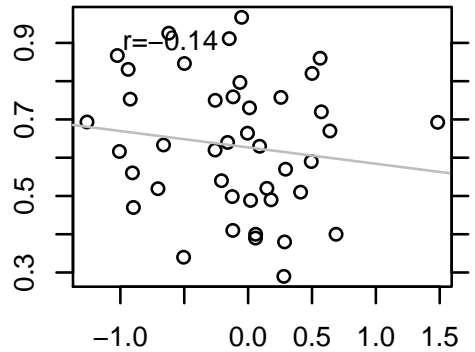

Relative Intensity [ $\log 10]$ 
M64 Glucose-6-phosphate

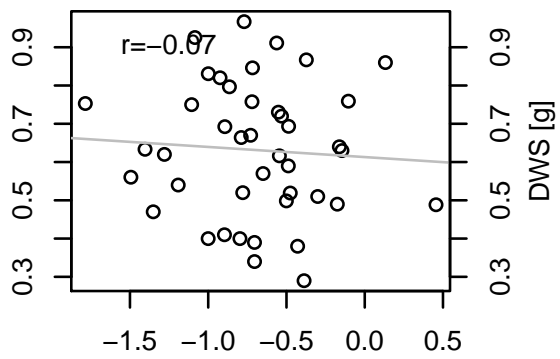

Relative Intensity [log10]

M65 MST 2360.1

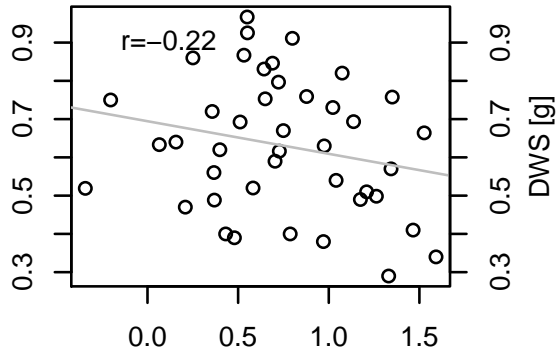

Relative Intensity [log10]

M66 MST 2482.9

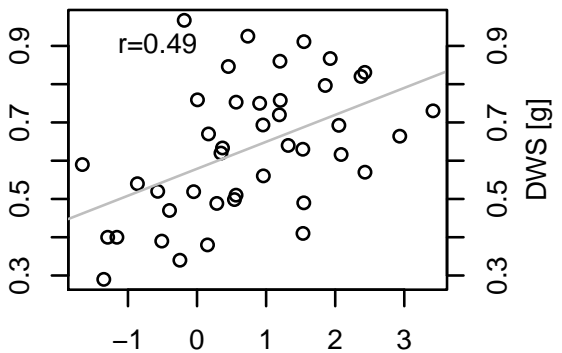

Relative Intensity [log10]
M67 Sugar Phosphate

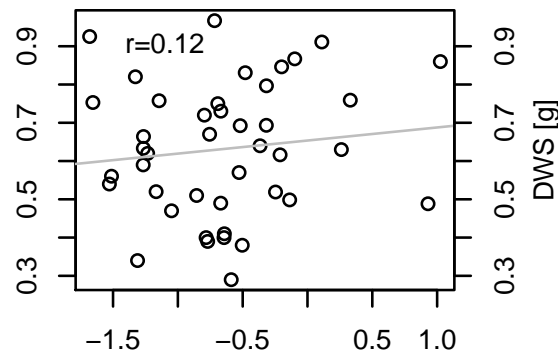

Relative Intensity [log10]

M68 Salicylic acid-glucopyranoside

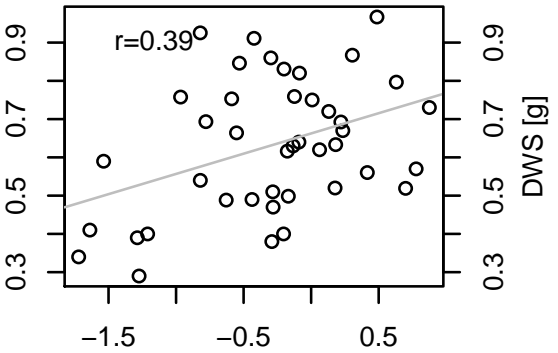

Relative Intensity [log10]

M69 MST 2578.73

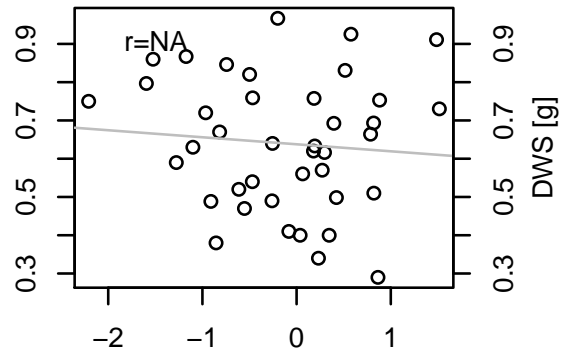

Relative Intensity [log10]
M70 Sucrose

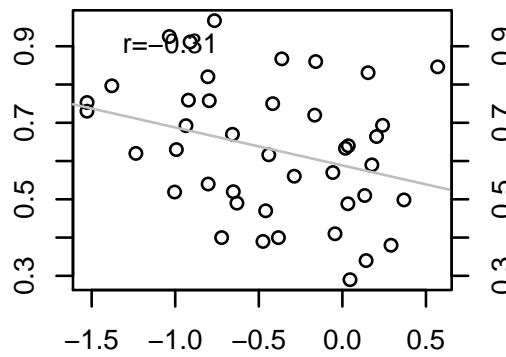

Relative Intensity [log10]

M71 MST 2656.76

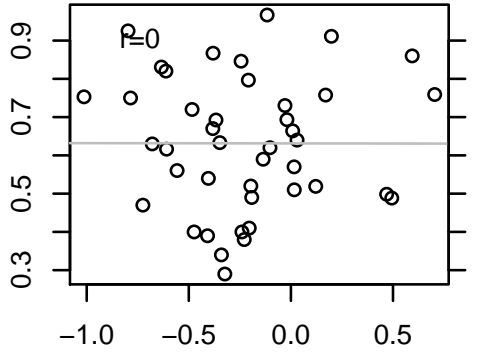

Relative Intensity [ $\log 10]$

M72 Lactose

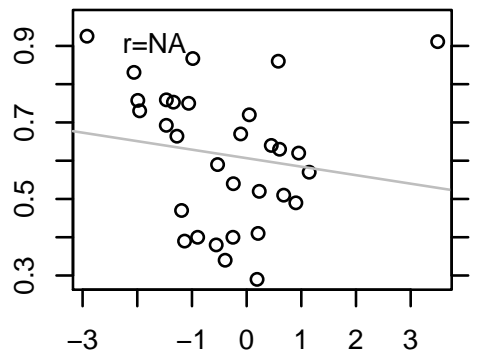

Relative Intensity [ $\log 10]$ 
M73 Trehalose

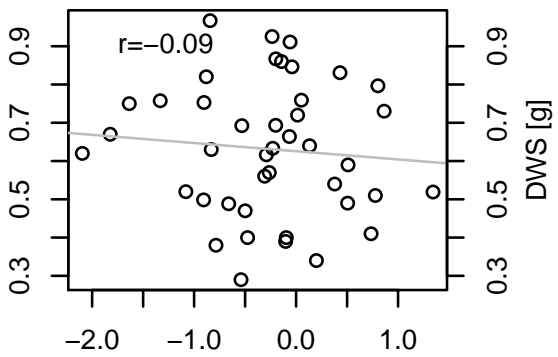

Relative Intensity [log10]
M76 MST 2913.73

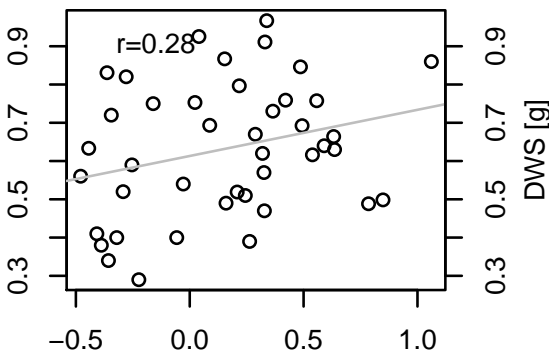

Relative Intensity [log10]
M79 Raffinose

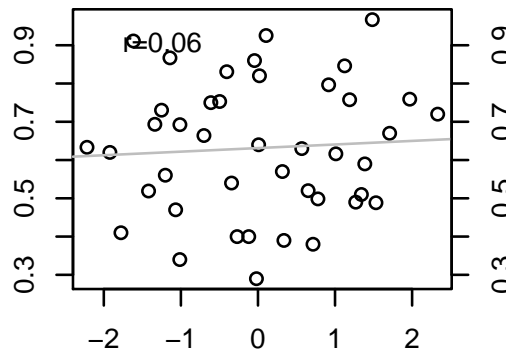

Relative Intensity [log10]
M74 MST 2866.46

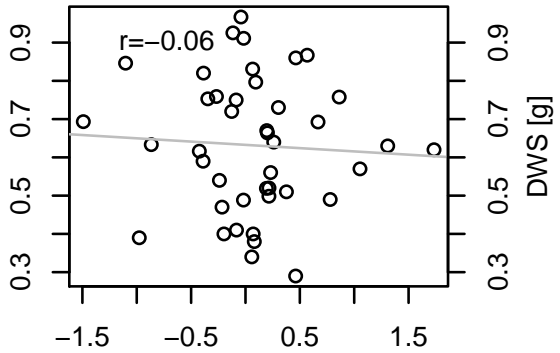

Relative Intensity [log10]

M75 Conjugate (Glycosyl, Inositol)

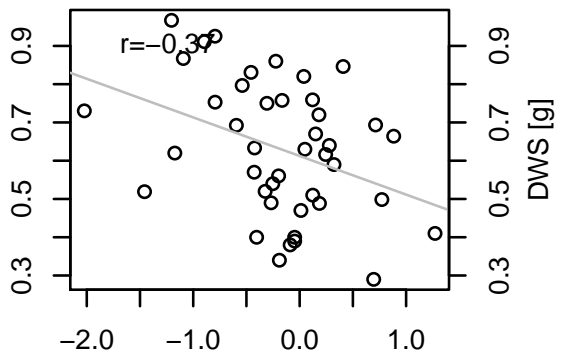

Relative Intensity [log10]
M77 Galactinol

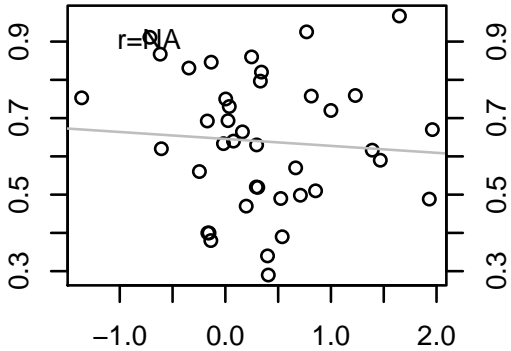

Relative Intensity [log10]

M78 MST 3092.87

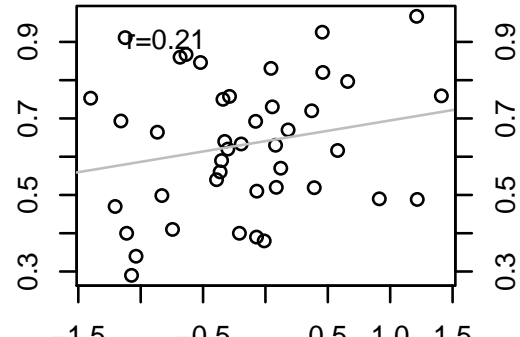

Relative Intensity [log10] 